\title{
Irregularities in the Distribution of Primes and Twin Primes
}

\author{
By Richard P. Brent
}

\begin{abstract}
The maxima and minima of $\langle L(x)\rangle-\pi(x),\langle R(x)\rangle-\pi(x)$, and $\left\langle L_{2}(x)\right\rangle-\pi_{2}(x)$ in various intervals up to $x=8 \times 10^{10}$ are tabulated. Here $\pi(x)$ and $\pi_{2}(x)$ are respectively the number of primes and twin primes not exceeding $x, L(x)$ is the logarithmic integral, $R(x)$ is Riemann's approximation to $\pi(x)$, and $L_{2}(x)$ is the Hardy-Littlewood approximation to $\pi_{2}(x)$. The computation of the sum of inverses of twin primes less than $8 \times 10^{10}$ gives a probable value $1.9021604 \pm 5 \times 10^{-7}$ for Brun's constant.
\end{abstract}

1. Approximations to $\pi(x)$. Let $P=\{2,3,5, \cdots\}$ be the set of primes, and let $\pi(x)$ be the number of primes not exceeding $x$. Two well-known approximations to $\pi(x)$ for $x>1$ are the logarithmic integral:

$$
\begin{aligned}
L(x) & =f_{0}^{x} \frac{d t}{\log t} \\
& =\gamma+\log (\log x)+\sum_{k=1}^{\infty} \frac{(\log x)^{k}}{k ! k}
\end{aligned}
$$

and Riemann's approximation:

$$
\begin{aligned}
R(x) & =\sum_{k=1}^{\infty} \frac{\mu(k)}{k} L\left(x^{1 / k}\right) \\
& =1+\sum_{k=1}^{\infty} \frac{(\log x)^{k}}{k ! k \zeta(k+1)} .
\end{aligned}
$$

Note that $(1.1)$ differs by $L(2)=1.04516378 \ldots$ from the frequently used approximation $\int_{2}^{x} d t / \log t$.

We are interested in the errors

and

$$
r_{1}(x)=\langle L(x)\rangle-\pi(x)
$$

$$
r_{2}(x)=\langle R(x)\rangle-\pi(x),
$$

where $\langle y\rangle$ denotes the integer closest to $y$ (i.e., the integer part of $(y+1 / 2))$.

Received July 5, 1974.

AMS (MOS) subject classifications (1970). Primary 10-04, 10H25, 10H15; Secondary 10A25, 10A40, 10H05, 65 A05, 65B05.

Key words and phrases. Prime, twin prime, Riemann's approximation, error bounds, Hardy-Littlewood conjecture, Brun's constant, logarithmic integral. 
Since $r_{i}(x)$ is usually (though not always: see below) of order $x^{1 / 2} / \log x$, it is useful to consider the "normalized" errors

$$
s_{i}(x)=r_{i}(x)(\log x) / x^{1 / 2} \quad \text { for } i=1,2 .
$$

Littlewood showed that, for sufficiently large $x, s_{i}(x) / \log \log \log x$ attains arbitrarily large positive and negative values [9], [10], [13]. On the other hand, Vinogradov [22] has shown that

$$
s_{i}(x)=O\left(x^{1 / 2} \exp \left(-\alpha(\log x)^{3 / 5}\right)\right)
$$

for a positive constant $\alpha$. Assuming the Riemann hypothesis, the stronger result

$$
s_{i}(x)=O\left(\log ^{2} x\right)
$$

is known [10]. Explicit bounds are given by Rosser and Schoenfeld [16]

Since $\pi(x)$ has been computed, both directly and indirectly [3] , [12] , [14], and tabulated for various values of $x$ up to $10^{13}$, the error functions $r_{i}(x)$ and $s_{i}(x)$ are easily computed for these values of $x$. However, Shanks [19] observed that this gives little information about the behaviour of the error functions between the tabulated values. Let

$$
R_{i}(a, b)=\max _{p \in P \cap[a, b]} r_{i}(p)
$$

and

$$
\rho_{i}(a, b)=\min _{p \in P \cap[a, b]} r_{i}(p) \text {. }
$$

In Section 4 we describe how $R_{i}(a, b)$ and $\rho_{i}(a, b)$ may be computed fairly efficiently for a given interval $[a, b]$. Table 1 gives the results of such computations for various intervals up to $8 \times 10^{10}$, and more detailed tables have been deposited in the UMT file of this journal. Although the maximum and minimum in (1.10) and (1.11) are taken only over primes in $[a, b]$, it is easy to see that

$$
\min _{x \in[a, b]} r_{i}(x)=\min \left(\rho_{i}(a, b), r_{i}(a)\right)
$$

and, except in the unlikely event that $r_{i}(x)$ does not have a jump at each prime in $[a, b]$,

$$
\max _{x \in[a, b]} r_{i}(x)=\max \left(R_{i}(a+1, b)+1, r_{i}(b)\right) .
$$

$s_{i}(x)$ oscillates so rapidly that it is difficult to plot it over any large domain of $x$ values. However, upper and lower bounds on $s_{i}(p)$ for primes $p \in[a, b]$ are easily found from (1.7), (1.10) and (1.11) once $R_{i}(a, b)$ and $\rho_{i}(a, b)$ are known. These bounds are fairly sharp if $b$ is close to $a$. Figure 1 shows such upper and lower bounds on $s_{2}(p)$, plotted against $\log _{10}((a+b) / 2)$, for various intervals $[a, b]$ which cover $\left[10^{4}, 8 \times 10^{10}\right]$ and satisfy $1.05 \leqslant b / a \leqslant 1.10$. The graph of upper and lower bounds on $s_{1}(p)$ looks similar since, from (1.3), 
TABLE 1

Extrema of approximation errors in $[a, b]$

\begin{tabular}{ccrrrrrr}
\hline$a$ & $b$ & $\rho_{1}$ & $R_{1}$ & $\rho_{2}$ & $R_{2}$ & $\rho_{3}$ & $R_{3}$ \\
\hline 2 & 10 & 0 & 1 & 0 & 1 & 1 & 1 \\
10 & $10^{2}$ & 1 & 4 & -1 & 0 & 2 & 4 \\
$10^{2}$ & $10^{3}$ & 3 & 10 & -2 & 1 & 3 & 9 \\
$10^{3}$ & $10^{4}$ & 7 & 23 & -6 & 5 & 3 & 12 \\
$10^{4}$ & $10^{5}$ & 13 & 54 & -16 & 13 & 5 & 41 \\
$10^{5}$ & $2 \times 10^{5}$ & 29 & 72 & -19 & 20 & 13 & 39 \\
$2 \times 10^{5}$ & $5 \times 10^{5}$ & 35 & 107 & -33 & 33 & 6 & 71 \\
$5 \times 10^{5}$ & $10^{6}$ & 50 & 135 & -36 & 35 & 37 & 97 \\
$10^{6}$ & $2 \times 10^{6}$ & 60 & 174 & -51 & 49 & -88 & 78 \\
$2 \times 10^{6}$ & $5 \times 10^{6}$ & 79 & 261 & -84 & 81 & -197 & -17 \\
$5 \times 10^{6}$ & $10^{7}$ & 118 & 346 & -98 & 95 & -280 & -44 \\
$10^{7}$ & $2 \times 10^{7}$ & 134 & 435 & -145 & 127 & -281 & -108 \\
$2 \times 10^{7}$ & $5 \times 10^{7}$ & 170 & 692 & -231 & 260 & -248 & 37 \\
$5 \times 10^{7}$ & $10^{8}$ & 344 & 895 & -242 & 260 & -29 & 262 \\
$10^{8}$ & $2 \times 10^{8}$ & 239 & 1149 & -514 & 336 & -143 & 643 \\
$2 \times 10^{8}$ & $5 \times 10^{8}$ & 585 & 1724 & -544 & 565 & 360 & 1046 \\
$5 \times 10^{8}$ & $10^{9}$ & 744 & 2668 & -685 & 965 & 536 & 1488 \\
$10^{9}$ & $2 \times 10^{9}$ & 770 & 3354 & -1093 & 982 & 566 & 2669 \\
$2 \times 10^{9}$ & $5 \times 10^{9}$ & 1316 & 4612 & -1681 & 1567 & -336 & 2130 \\
$5 \times 10^{9}$ & $10^{10}$ & 2129 & 7048 & -2387 & 2657 & -1930 & 696 \\
$10^{10}$ & $2 \times 10^{10}$ & 2159 & 10334 & -2776 & 3787 & -5833 & 2143 \\
\hline $5 \times 10^{10}$ & $5 \times 10^{10}$ & 3132 & 14990 & -4923 & 4950 & -7334 & 4443 \\
\hline & $8 \times 10^{10}$ & 5325 & 17065 & -5493 & 6106 & -2692 & 2846 \\
\hline
\end{tabular}

$$
s_{1}(x)=s_{2}(x)+1+O(1 / \log x)
$$

as $x \rightarrow \infty$.

The distribution of 11966 tabulated values of $s_{2}(n)$ for $n \in\left[10^{3}, 8.3 \times 10^{10}\right]$ is shown in Figure 2. The sample mean and standard deviation are 0.003 and 0.206 respectively. It is plausible to conjecture that a limiting distribution exists, with mean zero and standard deviation about 0.21 .

Some primes $p$ for which $\left|s_{2}(p)\right|$ is unusually large are given in Table 2. In fact, if an "exceptional peak" is a maximal interval $[a, b]$ such that $r_{2}(p)$ has constant sign for all primes $p$ in $(a, b)$, and $\left|s_{2}(p)\right| \geqslant 0.6$ for at least one prime $p$ in $(a, b)$, then Table 2 includes a prime $p$ (with maximal $\left|r_{2}(p)\right|$ ) from each exceptional peak in $\left[10^{4}, 8 \times 10^{10}\right]$. The entry $s_{1}(30909673)=0.52 \ldots$ was found by Appel and Rosser [1]. On the basis of Mapes' computations of $\pi\left(1.1 \times 10^{8}\right)$ and $\pi\left(1.8 \times 10^{8}\right)$, Shanks [19] conjectured that lower values of 


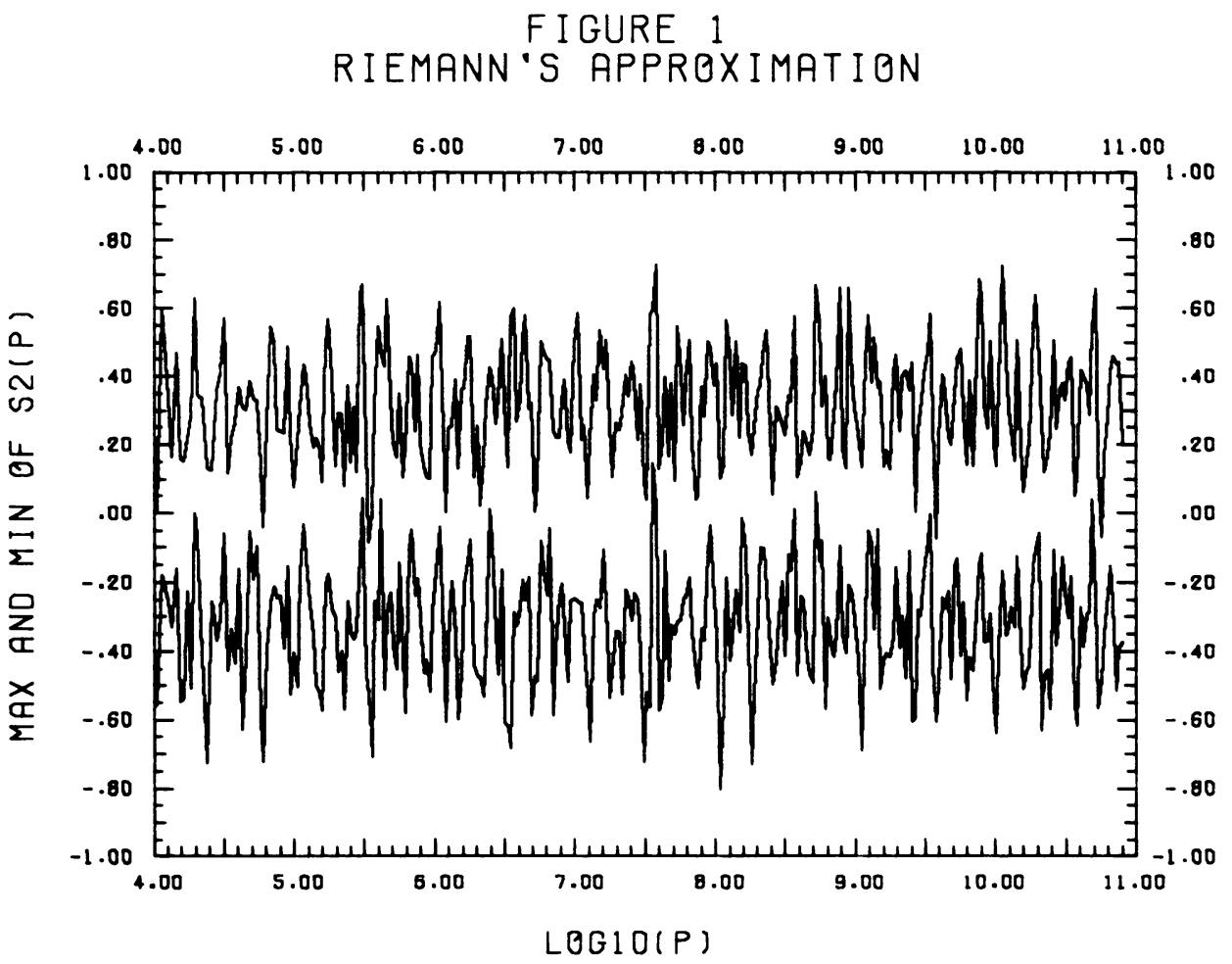

$s_{1}(p)$ could be found near $1.1 \times 10^{8}$ and $1.8 \times 10^{8}$, and the first and third entries in Table 2 show that this is correct.

Table 2 and an examination of the primes less than $10^{4}$ show that

$$
s_{1}(p)>0.42
$$

for all prime $p \in\left[5,8 \times 10^{10}\right]$, and hence

$$
\pi(x)<L(x)
$$

for $x \leqslant 8 \times 10^{10}$. This extends the result of Rosser and Schoenfeld [16], who proved (1.16) for $x \leqslant 10^{8}$. Note that $\left|r_{1}(p)\right|<\left|r_{2}(p)\right|$ for several entries in Table 2. The table also shows that

$$
-0.79<s_{2}(p)<0.75
$$

for all prime $p$ in $\left[10^{4}, 8 \times 10^{10}\right]$, and examination of primes less than $10^{4}$ then shows that

$$
-0.90<s_{2}(p)<0.75
$$

for all prime $p \leqslant 8 \times 10^{10}$.

Shanks [18] suggested the plausibility of

$$
\lim _{N \rightarrow \infty} \frac{1}{N} \sum_{n=2}^{N} s_{1}(n)=1
$$




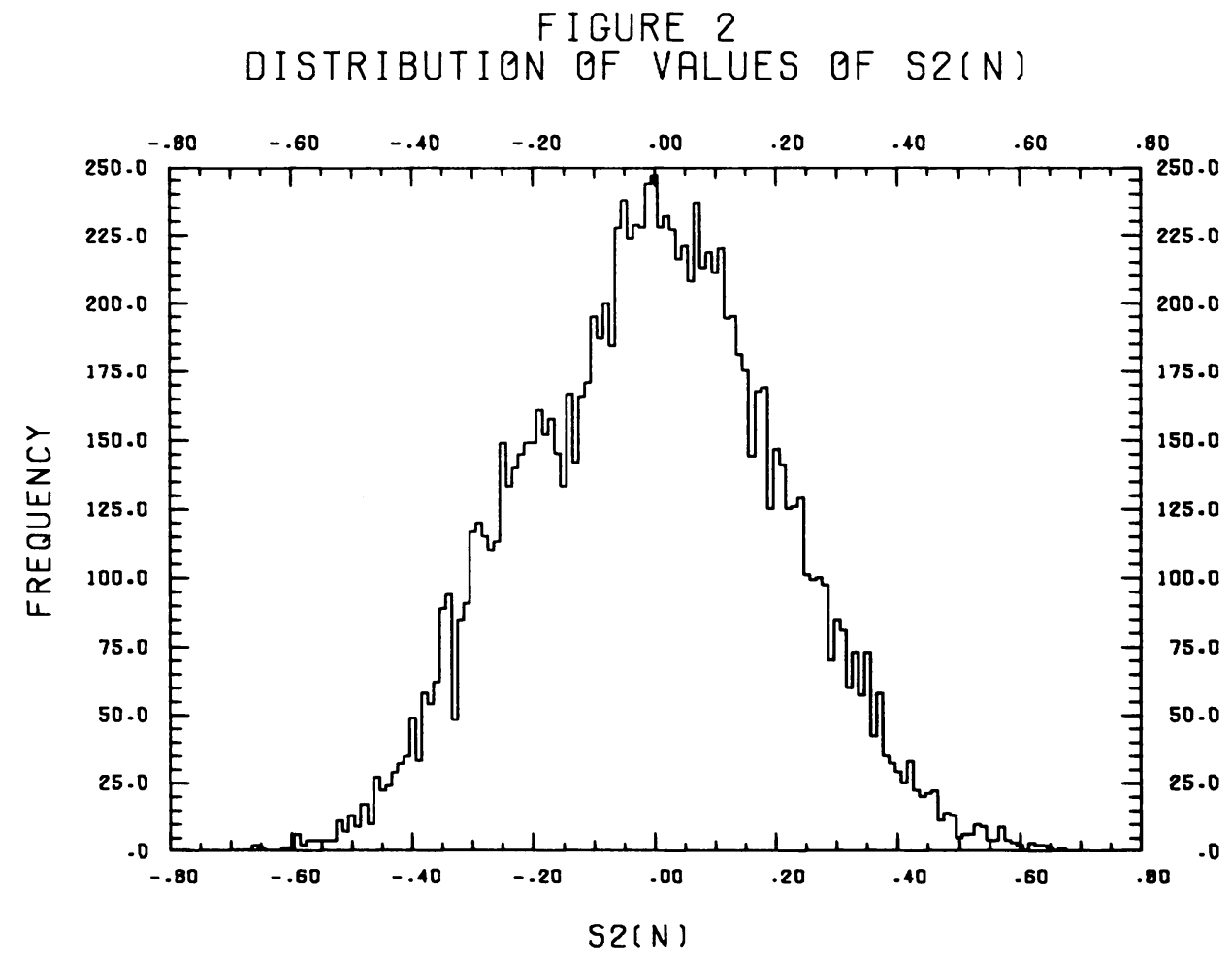

or, equivalently in view of (1.14),

$$
\lim _{N \rightarrow \infty} \frac{1}{N} \sum_{n=2}^{N} s_{2}(n)=0 .
$$

If true, (1.19) and (1.20) would give a sense in which Riemann's approximation (or even the simpler approximation $L(x)-1 / 2 L\left(x^{1 / 2}\right)$ obtained by taking the first two terms in (1.3)) is better than the logarithmic intergral approximation. However, Table 3 gives some evidence that the limits in (1.19) and (1.20) may not exist. If there are large intervals in which $s_{2}(n)$ is uniformly bounded away from zero and of constant sign, then (1.20) can hold only if the lengths of such intervals near $N$ are $o(N)$ as $N \rightarrow \infty$. Table 3 gives some disjoint intervals $[a, b]$ such that $10^{4} \leqslant b \leqslant 8 \times 10^{10}, b / a \geqslant 1.08$, and $r_{2}(p)$ has constant sign for all prime $p$ in $[a, b]$. The number of such intervals in each decade seems to be roughly constant. Intervals in which $\left|s_{2}(x)\right| \geqslant 0.01$ (say) are only slightly smaller than the intervals given in Table 3 .

The limit is more likely to exist if the mean of $s_{2}(n)$ is taken with respect to $\log n$ rather than $n$. This suggests the conjecture

$$
\lim _{N \rightarrow \infty}\left(\frac{1}{\log N}\right) \sum_{n=2}^{N} s_{2}(n) / n=0
$$


TABLE 2

Some primes $p$ with $\left|s_{2}(p)\right| \geqslant 0.6$

\begin{tabular}{|c|c|c|c|c|c|}
\hline$p$ & $\pi(p)$ & $r_{1}(p)$ & $r_{2}(p)$ & $s_{1}(p)$ & $s_{2}(p)$ \\
\hline 110102617 & 6308959 & 239 & -446 & 0.4218 & -0.7871 \\
\hline 36917099 & 2256804 & 692 & 260 & 1.9845 & 0.7456 \\
\hline 179845447 & 10022306 & 331 & -514 & 0.4691 & -0.7285 \\
\hline 11467849447 & 518601767 & 8594 & 3352 & 1.8589 & 0.7250 \\
\hline 59753 & 6041 & 19 & -16 & 0.8548 & -0.7199 \\
\hline 30909673 & 1910834 & 170 & -231 & 0.5274 & -0.7166 \\
\hline 24137 & 2688 & 14 & -11 & 0.9094 & -0.7145 \\
\hline 355111 & 30392 & 35 & -33 & 0.7506 & -0.7077 \\
\hline 7712599823 & 355168013 & 7048 & 2657 & 1.8271 & 0.6888 \\
\hline 302831 & 26218 & 93 & 30 & 2.1329 & 0.6880 \\
\hline 1110072773 & 56146451 & 770 & -1093 & 0.4813 & -0.6833 \\
\hline 3445943 & 246651 & 79 & -84 & 0.6406 & -0.6811 \\
\hline 516128797 & 27159319 & 2100 & 766 & 1.8544 & 0.6764 \\
\hline 50229461677 & 2128963733 & 16289 & 6106 & 1.7908 & 0.6713 \\
\hline 766449311 & 39507064 & 2489 & 905 & 1.8392 & 0.6687 \\
\hline 12871811 & 841519 & 134 & -145 & 0.6114 & -0.6616 \\
\hline 905055691 & 46254156 & 2668 & 965 & 1.8290 & 0.6615 \\
\hline 18834002419 & 832984013 & 10334 & 3787 & 1.7815 & 0.6529 \\
\hline 10016844407 & 455784972 & 2159 & -2776 & 0.4967 & -0.6387 \\
\hline 19373 & 2192 & 33 & 9 & 2.3405 & 0.6383 \\
\hline 463181 & 38685 & 107 & 33 & 2.0511 & 0.6326 \\
\hline 1090697 & 85021 & 151 & 47 & 2.0101 & 0.6257 \\
\hline 21728785387 & 954969014 & 3132 & -3850 & 0.5057 & -0.6217 \\
\hline 3278837 & 235526 & 84 & -75 & 0.6960 & -0.6214 \\
\hline 42863 & 4483 & 19 & -12 & 0.9788 & -0.6182 \\
\hline 38177961203 & 1637252682 & 4075 & -4923 & 0.5082 & -0.6139 \\
\hline 3593311 & 256264 & 242 & 77 & 1.9270 & 0.6131 \\
\hline 3745619057 & 178440671 & 1504 & -1681 & 0.5417 & -0.6055 \\
\hline 11777 & 1410 & 27 & 7 & 2.3322 & 0.6046 \\
\hline 1195247 & 92607 & 60 & -47 & 0.7680 & -0.6016 \\
\hline 10219591 & 678161 & 372 & 119 & 1.8781 & 0.6008 \\
\hline
\end{tabular}

or equivalently,

$$
\lim _{N \rightarrow \infty}\left(\frac{1}{\log N}\right) \sum_{n=2}^{N} s_{1}(n) / n=1
$$

Note that (1.20) implies (1.21), but not conversely. 
TABle 3

Some intervals $[a, b]$ where $r_{2}(p)$ has constant sign and $b / a \geqslant 1.08$

\begin{tabular}{rrrrr}
\hline \multicolumn{1}{c}{$a$} & $b$ & $b / a$ & $\rho_{2}(a, b)$ & $R_{2}(a, b)$ \\
\hline 9278 & 11046 & 1.191 & -6 & 0 \\
45894 & 49942 & 1.088 & 0 & 8 \\
56478 & 62850 & 1.113 & -16 & 0 \\
164912 & 179748 & 1.090 & 0 & 20 \\
291570 & 318916 & 1.094 & 0 & 30 \\
324090 & 369790 & 1.141 & -33 & 0 \\
638372 & 689958 & 1.081 & 0 & 28 \\
4889994 & 5530998 & 1.131 & -84 & 0 \\
6862134 & 7472358 & 1.089 & -98 & 0 \\
9867492 & 10673698 & 1.082 & 0 & 119 \\
34225760 & 38856760 & 1.135 & 0 & 260 \\
504454344 & 552984016 & 1.096 & 0 & 766 \\
3219006864 & 3507922926 & 1.090 & 0 & 1567 \\
3637747892 & 4013111982 & 1.103 & -1681 & 0 \\
35699734892 & 38858023776 & 1.088 & -4923 & 0 \\
47048490524 & 51040905052 & 1.085 & 0 & 6106 \\
53087472258 & 58483092228 & 1.102 & -5288 & 0 \\
\hline
\end{tabular}

Let us return to the conjecture of a limiting distribution for $s_{2}(x)$. The above discussion shows that care must be taken in formalizing the conjecture, for if $x$ and $y$ are drawn from $[a, b]$, then $s_{2}(x)$ and $s_{2}(y)$ will certainly be dependent if $b / a$ is too close to 1 . One possibility is to conjecture that the sequence $\left(s_{2}\left(x_{i}\right)\right)$ has a limiting distribution if $\left(x_{i}\right)$ is a random sequence of positive numbers such that $x_{i} / x_{i+1} \rightarrow 0$ (and hence $x_{i} \rightarrow \infty$ ) as $i \rightarrow \infty$.

If the conjecture is true, and if the limiting distribution is approximately normal, with mean 0 and standard deviation about 0.21 , we would expect $s_{2}(x)<$ -1 (or $s_{1}(x)<0$ ) for about one in every $10^{6}$ independent random samples. Similarly, we would expect $s_{2}(x)<-0.6$ for about one in 450 independent samples. Since Table 2 covers the range $4.0 \leqslant \log _{10} x \leqslant 10.9$, and includes 17 entries with $s_{2}(x)<-0.6$, we would expect an entry with $s_{2}(x)<-1$ if the table could be extended to about

$$
\log _{10} x=\left(\frac{10.9-4.0}{17}\right)\left(\frac{10^{6}}{450}\right) \simeq 900
$$

Although this argument is very crude, it suggests that (1.16) probably holds for $\log _{10} x$ up to about 100 (well beyond the range of feasible computation). It is 
known that (1.16) is violated long before the legendary Skewes' number [21]; specifically, Lehman proved [11] that certain integers $x$ between $1.53 \times 10^{1165}$ and $1.65 \times 10^{1165}$ suffice.

2. Approximations to $\pi_{2}(x)$. We say that $q$ is a "twin prime" if both $q$ and $q+2$ are prime. Let $Q=\{3,5,11,17, \ldots\}$ be the set of twin primes, and let $\pi_{2}(x)$ be the number of twin primes not exceeding $x$. The Hardy-Littlewood approximation to $\pi_{2}(x)$ is

$$
L_{2}(x)=2 c_{2} \int_{2}^{x} \frac{d t}{\log ^{2} t}
$$

where

$$
c_{2}=\prod_{2<p \in P} \frac{1-2 / p}{(1-1 / p)^{2}}=0.66016181 \ldots
$$

is the "twin-prime" constant [24].

Properties of $\pi(x)$ may be proved using the well-known relationship between the distribution of primes and the location of the zeros of the Riemann zeta function [10, Chapter 4]. Unfortunately, no similar relationship is known for twin primes, so very little is known about $\pi_{2}(x)$. It is not known whether there are infinitely many twin primes, and much less whether

$$
\pi_{2}(x) \sim L_{2}(x)
$$

as $x \longrightarrow \infty$. However, empirical evidence suggests that (2.3) is true. In Table 4 we give $\pi_{2}(n)$ and

$$
r_{3}(n)=\left\langle L_{2}(n)\right\rangle-\pi_{2}(n)
$$

for various $n \leqslant 8 \times 10^{10}$. The values of $\pi_{2}(n)$ were computed by enumerating the primes up to $n$ and counting the number of twins, for no more subtle method is known. Our counts agree with those of Weintraub [23] (for $n \leqslant 2 \times 10^{8}$ ) and Bohman [4] (for $n \leqslant 2 \times 10^{9}$ ).

Let

$$
R_{3}(a, b)=\max _{q \in \bigcap \cap[a, b]} r_{3}(q)
$$

and

$$
\rho_{3}(a, b)=\min _{q \in Q \cap[a, b]} r_{3}(q) .
$$

The functions $R_{3}(a, b)$ and $\rho_{3}(a, b)$ were computed for various intervals $[a, b]$ up to $8 \times 10^{10}$, and some results are given in Table 1 . More detailed tables have been deposited in the UMT file of this journal. 
TABLE 4

Counts of twin primes and estimates of Brun's constant

\begin{tabular}{|c|c|c|c|c|}
\hline$n$ & $\pi_{2}(n)$ & $r_{3}(n)$ & $B(n)$ & $B^{*}(n)$ \\
\hline $10^{3}$ & 35 & 11 & 1.518032463560 & 1.90030531 \\
\hline $10^{4}$ & 205 & 9 & 1.616893557432 & 1.90359819 \\
\hline $10^{5}$ & 1224 & 25 & 1.672799584828 & 1.90216329 \\
\hline $10^{6}$ & 8169 & 79 & 1.710776930804 & 1.90191335 \\
\hline $10^{7}$ & 58980 & -226 & 1.738357043917 & 1.90218826 \\
\hline $10^{8}$ & 440312 & 56 & 1.758815621068 & 1.90216794 \\
\hline $10^{9}$ & 3424506 & 802 & 1.774735957639 & 1.90216024 \\
\hline $2 \times 10^{9}$ & 6388041 & 984 & 1.778859404547 & 1.90215957 \\
\hline $3 \times 10^{9}$ & 9210144 & 461 & 1.781150604842 & 1.90215977 \\
\hline $4 \times 10^{9}$ & 11944438 & 1032 & 1.782724861607 & 1.90215950 \\
\hline $5 \times 10^{9}$ & 14618166 & 291 & 1.783918570267 & 1.90215984 \\
\hline $6 \times 10^{9}$ & 17244409 & -770 & 1.784876490721 & 1.90216027 \\
\hline $7 \times 10^{9}$ & 19830161 & -119 & 1.785673823717 & 1.90216007 \\
\hline $8 \times 10^{9}$ & 22384176 & -248 & 1.786355995279 & 1.90216011 \\
\hline $9 \times 10^{9}$ & 24911210 & -1324 & 1.786951346213 & 1.90216037 \\
\hline $10^{10}$ & 27412679 & -1262 & 1.787478502719 & 1.90216036 \\
\hline $2 \times 10^{10}$ & 51509099 & -4667 & 1.790830284135 & 1.90216076 \\
\hline $3 \times 10^{10}$ & 74555618 & -3348 & 1.792701319111 & 1.90216064 \\
\hline $4 \times 10^{10}$ & 96956707 & 1869 & 1.793990899123 & 1.90216031 \\
\hline $5 \times 10^{10}$ & 118903682 & 1630 & 1.794970693076 & 1.90216031 \\
\hline $6 \times 10^{10}$ & 140494397 & 1555 & 1.795758170053 & 1.90216033 \\
\hline $7 \times 10^{10}$ & 161795029 & 2031 & 1.796414982022 & 1.90216032 \\
\hline $8 \times 10^{10}$ & 182855913 & -985 & 1.796977508288 & 1.90216040 \\
\hline
\end{tabular}

Let $s_{3}(x)$ be defined by (1.7) with $i=3$. Upper and lower bounds on $s_{3}$ in various intervals were computed in the same way as for $s_{2}$, and are shown in Figure 3. Comparison of Figures 1 and 3 shows that the behaviour of $s_{3}$ is quite different from that of $s_{2}$ (or $s_{1}$ ). Although $s_{3}(q)$ changes sign, there are large intervals in which it is of constant sign. For example, $s_{3}(q)$ is positive for all twin primes $q$ in $\left[3,1.36 \times 10^{6}\right]$, negative in $\left[1.52 \times 10^{6}, 3.52 \times 10^{7}\right]$, positive in $\left[1.50 \times 10^{8}, 3.06 \times 10^{9}\right]$, negative in $\left[1.19 \times 10^{10}, 2.71 \times 10^{10}\right]$, etc. Hence, it seems unlikely that the limit corresponding to (1.19) exists, although it is possible that the limit corresponding to (1.22) (with $s_{1}$ replaced by $s_{3}$ ) exists.

Suppose that the integers $4,5, \cdots, N$ are randomly and independently selected or rejected, with the probability of selection of $n$ being $2 c_{2} / \log ^{2} n$. If 


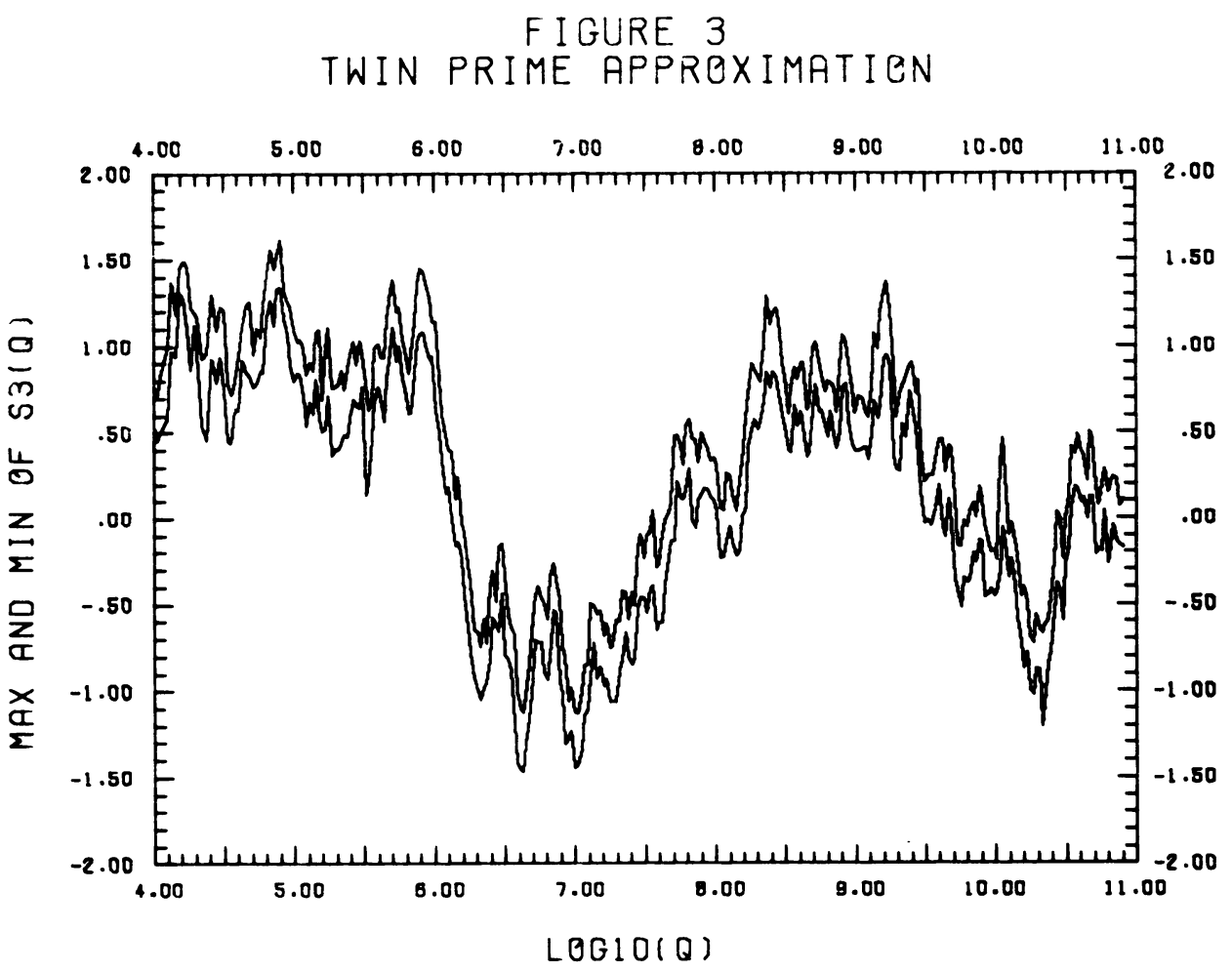

$P(N)$ is the number of integers selected, then $P(N)$ is distributed with mean $\mu(N)$ $\sim L_{2}(N)$ and variance $\sigma^{2}(N) \sim L_{2}(N)$, and the distribution is asymptotically normal as $N \rightarrow \infty$. Thus $S(N)=\left(L_{2}(N)-P(N)\right)(\log N) / N^{1 / 2}$ is asymptotically normal with mean zero and standard deviation $\left(2 c_{2}\right)^{1 / 2} \bumpeq 1.15$. It is interesting to note that $\pi_{2}(N)$ and $s_{3}(N)$ appear to behave like $P(N)$ and $S(N)$ respectively. (The analogy for primes is apparently false, for it predicts that $s_{1}(N)$ should have mean 0 and standard deviation $O\left((\log N)^{1 / 2}\right)$, and does not predict the frequent fluctuations in $s_{1}(N)$ (compare Figures 1 and 3 ). For some rigorous results connecting primes with random walks, see [2].)

We shall briefly mention some other approximations to $\pi_{2}(x)$. The simplest is $2 c_{2} x / \log ^{2} x$, which differs from $L_{2}(x)$ by terms of order $x / \log ^{3} x$. The empirical results discussed above show that

$$
\left|s_{3}(q)\right|<2.3
$$

for all twin primes $q \leqslant 8 \times 10^{10}$, so $\left|L_{2}(x)-\pi_{2}(x)\right|$ is of order $x^{1 / 2} / \log x$ for $x \leqslant 8 \times 10^{10}$. Hence, $L_{2}(x)$ is a more accurate approximation, at least in the range considered.

Other approximations are obtained by replacing $1 / \log ^{2} t$ in $(2.1)$ by $\left(R^{\prime}(t)\right)^{2}$ or by $\left(2 R^{\prime}(t) / \log t-1 / \log ^{2} t\right)$, as suggested by Fröberg [8] and Shanks and Wrench [20], respectively. Since these approximations differ from $L_{2}(x)$ by terms of order 
$x^{1 / 2} / \log ^{2} x$ they are not appreciably better or worse than $L_{2}(x)$ over most of the range $x \leqslant 8 \times 10^{10}$. The advantage of $L_{2}(x)$ is that it is easy to compute, e.g., from

$$
L_{2}(x)=2 c_{2}(L(x)+K-x / \log x)
$$

where $K=2 / \log 2-L(2)=1.84022630 \cdots$.

3. Brun's Constant. Let

$$
B(x)=\sum_{x \geqslant q \in 2}\left(\frac{1}{q}+\frac{1}{q+2}\right) .
$$

Brun [7] showed that "Brun's constant" $B(\infty)=\lim _{x \rightarrow \infty} B(x)$ is finite (although the sum of reciprocals of primes has been known to be infinite since Euler's time). We have followed the definition of Shanks and Wrench [20], although Brun [7] and Selmer [17] consider $B(\infty)-(1 / 3+1 / 5)$, and Bohman [4] considers $B(\infty)-1 / 5$.

Assuming that twin primes are distributed randomly with density $L_{2}^{\prime}(x)=$ $2 c_{2} / \log ^{2} x$ (see Section 2), we can estimate

$$
B(\infty)-B(x) \simeq 4 c_{2} \int_{x}^{\infty} \frac{d t}{t \log ^{2} t}=4 c_{2} / \log x,
$$

which suggests the definition

$$
B^{*}(x)=B(x)+4 c_{2} / \log x
$$

Although $\lim _{x \rightarrow \infty} B^{*}(x)=\lim _{x \rightarrow \infty} B(x)=B(\infty)$, it is probable that the rate of convergence of $B^{*}(x)$ is much faster than that of $B(x)$. In fact, in contrast to (3.2), we expect that $B^{*}(x)-B(\infty)$ is asymptotically normally distributed with mean $o\left(1 /\left(x^{1 / 2} \log x\right)\right)$ and standard deviation $\sim\left(8 c_{2}\right)^{1 / 2} /\left(x^{1 / 2} \log x\right)$.

Selmer [17] estimated $B(\infty)=1.901 \pm 0.014$ by extrapolation from $B(200000)$. Fröberg [8] computed $B(n)$ for several $n \leqslant 2^{20}$ and estimated $B(\infty)$ $=1.90195 \pm 3 \times 10^{-5}$. Shanks and Wrench [20] found $B(32452843)$ and estimated $B(\infty)=1.90218 \pm 2 \times 10^{-5}$. Finally, Bohman [4] computed $B\left(2 \times 10^{9}\right)$ and estimated $B(\infty)=1.90216 \pm 5 \times 10^{-6}$. During the computation of $\pi_{2}(n)$ as described above, we computed $B(n)$ and $B^{*}(n)$ for various $n \leqslant 8 \times 10^{10}$. Some values are given in Table 4 , and more are given in a table deposited in the UMT file of this journal. From our computation of $B^{*}\left(8 \times 10^{10}\right)$ we estimate that $B(\infty)$ probably lies in the range

$$
B(\infty)=1.9021604 \pm 5 \times 10^{-7} .
$$

In the computation of $B(n)$ we used floating-point arithmetic with a 60-bit fraction, and accumulated the sum using Moller's "quasi double-precision" device [15]. Hence, rounding errors should not affect the entries in Table 4. (Our values of $B(n)$ 
differ from Bohman's (corrected) values in the 9th decimal place, possibly because of the effect of rounding errors in his calculations.)

Although we do not know how to bound the error in our estimate (3.4), the discussion above suggests that $x^{1 / 2} \log x\left(B^{*}(x)-B(\infty)\right)$ is asymptotically normally distributed, and we certainly have

$$
\left|x^{1 / 2} \log x\left(B^{*}(x)-1.9021604\right)\right|<3.5
$$

for all tabulated values in the range $\left[10^{4}, 8 \times 10^{10}\right]$. (The maximum value of 3.4927 is at $x=860000$, in the region of the sharp drop in Figure 3.) Hence, it is probable that

$$
\left|B^{*}\left(8 \times 10^{10}\right)-B(\infty)\right|<\frac{3.5}{\left(8 \times 10^{10}\right)^{1 / 2} \log \left(8 \times 10^{10}\right)}<5 \times 10^{-7},
$$

which explains the error estimate in (3.4). If the constant $\left(8 c_{2}\right)^{1 / 2}$ above is correct, the probability that $B(\infty)$ is in the range given by (3.4) is about 0.88 .

Different methods of extrapolating $B(x)$ to the limit have been suggested by Fröberg [8] and Shanks and Wrench [20], but their extrapolations differ from $B^{*}(x)$ by $O\left(1 / x^{1 / 2} \log ^{2} x\right)$, so are probably not much better or worse than $B^{*}(x)$. It seems difficult to obtain an appreciably better extrapolation than $B^{*}(x)$ without being able to predict the large-scale oscillations of $s_{3}(x)$ (see Figure 3).

4. Computation of $R_{i}(a, b)$ and $\rho_{i}(a, b)$. If $R_{i}$ and $\rho_{i}$ are defined by (1.10) and (1.11), the most time-consuming part of their computation is not the generation of the primes in $[a, b]$, which may be done efficiently by a sieve method (as in [5], [6]), but the frequent evaluation of $L(x)$ and $R(x)$ to a precision sufficient to determine $\langle L(x)\rangle$ and $\langle R(x)\rangle$. (Similarly for $R_{3}$ and $\rho_{3}$ defined by (2.5) and (2.6), although the situation is not so clear here, because it takes longer, on the average, to generate a twin prime than a prime.)

To avoid evaluating $\langle L(p)\rangle$ and $\langle R(p)\rangle$ for every prime $p$ in $[a, b]$, we can use simple upper and lower bounds for $L(p)$ and $R(p)$, and only evaluate $\langle L(p)\rangle$ and $\langle R(p)\rangle$ if the upper and lower bounds fail to show that $r_{i}(p)$ lies within the maxima and minima already found. The following lemmas indicate how suitable upper and lower bounds may be found.

Lemma 1. Suppose $f^{\prime \prime}(x)<0$ on $[a, b]$, and $a<a^{\prime}<x<x^{\prime}<b^{\prime}<b$. Then

$$
\frac{f\left(a^{\prime}\right)-f(a)}{a^{\prime}-a}>\frac{f\left(x^{\prime}\right)-f(x)}{x^{\prime}-x}>\frac{f\left(b^{\prime}\right)-f(b)}{b^{\prime}-b} .
$$

The proof is immediate from a mean value theorem.

Lemma 2. $L^{\prime \prime}(x), R^{\prime \prime}(x)$ and $L_{2}^{\prime \prime}(x)$ are negative for $x>1$.

Proof. From (1.1), (2.1) and (1.4) we have $L^{\prime \prime}(x)=-1 /\left(x \log ^{2} x\right)<0, L_{2}^{\prime \prime}(x)=$ $-4 c_{2} /\left(x \log ^{3} x\right)<0$, and 


$$
R^{\prime \prime}(x)=-x^{-2} \sum_{k=1}^{\infty}\left(\frac{k+1}{\zeta(k+1)}-\frac{k}{\zeta(k+2)}\right) \frac{(\log x)^{k-1}}{(k+1) !}
$$

Now $\zeta(k+1)<1+1 / k$ for $k \geqslant 1$, so $(k+1) / \zeta(k+1)>k / \zeta(k+2)$, and the result follows from (4.1).

Acknowledgement. I am indebted to Daniel Shanks for acquainting me with reference [11] and making several very fruitful suggestions.

Computer Centre

Australian National University

Canberra, Australia

1. K. I. APPEL \& J. B. ROSSER, Table for Functions of Primes, IDA-CRD Technical Report Number 4, 1961 ; reviewed in RMT 55, Math. Comp., v. 16, 1962, pp. 500-501.

2. P. BILlingSLEY, "Prime numbers and Brownian motion," Amer. Math. Monthly, v. 80, 1973, pp. 1099-1115.

3. J. BOHMAN, "On the number of primes less than a given limit," Nordisk Tidskr. Informationsbehandling (BIT), v. 12, 1972, pp. 576-577.

4. J. BOHMAN, "Some computational results regarding the prime numbers below 2,000,000,000," Nordisk Tidskr. Informationsbehandling (BIT), v. 13, 1973, pp. 242-244; Errata, ibid., v. 14, 1974, p. 127.

5. R. P. BRENT, "The first occurrence of large gaps between successive primes,"Math. Comp., v. 27, 1973, pp. 959-963.

6. R. P. BRENT, "The distribution of small gaps between successive primes," Math. Comp., v. 28, 1974, pp. 315-324.

7. V. BRUN, "La série $1 / 5+1 / 7+1 / 11+1 / 13+1 / 17+1 / 19+1 / 29+1 / 31+1 / 41+$ $1 / 43+1 / 59+1 / 61+\ldots$, où les dénominateurs sont 'nombres premiers jumeaux' est convergente ou finie," Bull. Sci. Math., v. 43, 1919, pp. 124-128.

8. C.-E. FRÖBERG, "On the sum of inverses of primes and twin primes," Nordisk Tidskr. Informationsbehandling (BIT), v. 1, 1961, pp. 15-20.

9. G. H. HARDY \& J. E. LITTLEWOOD, "Contributions to the theory of the Riemann zeta function and the theory of the distribution of primes," Acta Math., v. 14, 1918, p. 127.

10. A. E. INGHAM, The Distribution of Prime Numbers, Cambridge Tract, no. 30, Cambridge Univ. Press, New York, 1932.

11. R. SHERMAN LEHMAN, “On the difference $\pi(x)-1 \mathrm{l}(x)$," Acta Arith., v. 11,1966 , pp. 397-410. MR 34 \#2546.

12. D. H. LEHMER, "On the exact number of primes less than a given limit," Illinois J. Math., v. 3, 1959, pp. 381-388. MR 21 \#5613.

13. J. E. LITTLEWOOD, "Sur la distribution des nombres premiers," Comptes Rendus, v. 158,1914 , pp. 263-266.

14. D. C. MAPES, "Fast method for computing the number of primes less than a given limit," Math. Comp., v. 17, 1963, pp. 179-185.

15. O. M $\emptyset L L E R$, "Quasi double-precision in floating point addition," Nordisk Tidskr. Informationsbehandling $(B I T)$, v. 5, 1965, pp. 37-50; Comment, ibid., v. 5, 1965, pp. 251-255. MR 31 \#5359.

16. J. B. ROSSER \& L. SCHOENFELD, "Approximate formulas for some functions of prime numbers," Illinois J. Math., v. 6, 1962, pp. 64-94; reviewed in RMT 40, Math. Comp., v. 17, 1963, pp. 307-308. MR 25 \#1139.

17. E.S. SELMER, "A special summation method in the theory of prime numbers and its application to 'Brun's sum'," Nordisk Mat. Tidskr., v. 24, 1942, pp. 74-81. (Norwegian) MR 8, 316.

18. D. SHANKS, "Quadratic residues and the distribution of primes," Math. Tables Aids Comput., v. 13, 1959, pp. 272-284. MR $21 \# 7186$. 
19. D. SHANKS, UMT 39, Math. Comp., v. 17, 1963, p. 307.

20. D. SHANKS \& J. W. WRENCH, JR., "Brun's constant," Math Comp., v. 28, 1974, pp. 293-299; Corrigendum, ibid., v. 28, 1974, p. 1183.

21. S. SKEWES, “On the difference $\pi(x)-\mathrm{li}(x)$. II," Proc. London Math. Soc. Ser. (3), v. 5, 1955, pp. 48-70. MR 16,676.

22. I. M. VINOGRADOV, "A new estimate of the function $\zeta(1+i t)$," Izv. Akad. Nauk SSSR Ser. Mat., v. 22, 1958, pp. 161-164. (Russian) MR 21 \#2624.

23. S. WEINTRAUB, UMT 38, Math. Comp., v. 27, 1973, pp. 676-677.

24. J. W. WRENCH, JR., "Evaluation of Artin's constant and the twin-prime constant," Math. Comp., v. 15, 1961, pp. 396-398. MR 23 \#A1619. 